\title{
AU Pegasi revisited: period evolution and orbital elements of a peculiar Type II Cepheid
}

\author{
Géza Csörnyei ${ }^{1,2}$ (1) - László Szabados ${ }^{1,3}$
}

Received: 16 July 2019 / Accepted: 3 September 2019 / Published online: 9 September 2019

(C) The Author(s) 2019

\begin{abstract}
New analysis on the period changes of Type II Cepheid AU Peg is presented. The available recent photometric measurements were collected and analysed with various methods. The period has been found to be constant for certain time intervals, although increasing in overall, in contrast with the previous expectations, which suggested the period change to reverse. Superimposed on overall period change, a formerly unknown periodic behaviour has been found in the $O-C$ diagram of AU Peg, which cannot be matched to the radial velocity variations. Since the Cepheid is a member of a binary system, it is probable that the unusual period change is in connection with the companion's tidal force. The orbital elements of the binary system involving AU Peg have been also revised.
\end{abstract}

Keywords Type II Cepheids · AU Peg · Period changes · Binary

\section{Introduction}

AU Pegasi is a Type II Cepheid with a pulsation period of approximately 2.4 days and with a mean spectral type of F8. This Cepheid is considered unique in several ways; most

$凶$ G. Csörnyei

csornyei.geza@csfk.mta.hu

1 Konkoly Observatory, Research Centre for Astronomy and Earth Sciences, Hungarian Academy of Sciences, Konkoly Thege Miklós út 15-17, 1121 Budapest, Hungary

2 Eötvös Loránd University, Institute of Physics, Pázmány Péter sétány 1/a, Budapest 1117, Hungary

3 MTA CSFK Lendület Near-Field Cosmology Research Group, Budapest, Hungary importantly it has been found to have a physical companion (Harris et al. 1979) and a highly unstable pulsation period (Szabados 1977; Erleksova 1978). The orbital period of this spectroscopic binary system is $\mathbf{5 3 . 3}$ days, which is the second shortest among the known binaries with a Type II Cepheid component. The only Type II Cepheid in a binary system with a shorter orbital period is TYC 1031012621 $\left(P_{\text {orb }}=51.38\right.$ days) (Antipin et al. 2007), while the other Galactic Type II Cepheids in binary systems, IX Cas and TX Del have an orbital period of 110.29 and 130.15 days, respectively (Harris and Welch 1989).

Harris et al. (1984) found that the colour of AU Pegasi is unusually red, which would be normal for a Cepheid with a longer pulsation period. The effective temperature of its atmosphere is remarkably low, $T_{\text {eff }}=5500-6000 \mathrm{~K}$, depending on the pulsational phase (Kovtyukh et al. 2018). Recent spectroscopic measurements revealed that the $[\mathrm{Fe} / \mathrm{H}]$ abundance ratio is 0.27 , which means that AU Peg is a metal rich Type II Cepheid (Kovtyukh et al. 2018).

The infrared excess and the unusual colour index $(B-$ $V=0.85$, Harris et al. 1984; Wallerstein 2002) also indicate the presence of a dust cloud surrounding the binary system (McAlary and Welch 1986). It has also been observed that the spectrum of this Cepheid exhibits P Cygni like behaviour, narrow emission features on the red side of the $\mathrm{H} \alpha$ line, which show variations on orbital period timescale, thus might be a result of interaction between the atmosphere of the star with the circumstellar matter around it (Vinkó et al. 1998). Presently it is thought that AU Pegasi is close to filling its Roche lobe and mass transfer between the two stars almost certainly occurred formerly (Maas et al. 2007).

The temporal behaviour of the pulsation period of AU Pegasi was extensively studied by Vinkó et al. (1993) in the time interval of JD 2433 100-2 448600. They found that the pulsation period was slightly increasing with a rate of 
$d P / d t=5 \cdot 10^{-7}$ day/day before JD 2440000 , while the average pulsation period length was $P_{\text {pul }}=2.39$ days. According to their study the first epoch where the rate of the period variation changed was between JD 2439000 and 2441000 . At this epoch the rate of the variation accelerated to $d P / d t=1.8 \cdot 10^{-6}$ day/day. This period variation has eventually seemed to stop between JD 2446700 and 2447800 . After this second break point the period variation seemed to reverse and to start decreasing.

They concluded that the rapid period change might be the result of the interaction between the variable star and its companion, but as they pointed out, the period variation cannot be explained by tidal interaction alone. Since the classification of Type II Cepheid is somewhat uncertain, it also had been suggested, that this variable star is a classical Cepheid crossing the instability strip for the first time (Vinkó et al. 1993). This could explain the rapid period variation, but the latest studies still classified this variable as a Type II Cepheid (Groenewegen 2018), which classification is also supported by the kinematics, the position of the star on the colour-magnitude diagram and length of the orbital period, as well.

The latest Gaia parallax of the object is $\Pi=1.6739 \pm$ 0.0448 milliarcsecond (Gaia Collaboration 2016, 2018), while the $V$-band extinction of the star is $A_{V}=0.184 \mathrm{mag}$ (NASA/IPAC Infrared Science Archive), which together correspond to an absolute magnitude of $M_{V}=0.069$ mag. According to the classical Cepheid period-luminosity relation (Benedict et al. 2007) this would correspond to a period of 0.214 days, which is significantly smaller than the one we observed, thus it supports the classification of the star as a Type II Cepheid.

In view of its importance and peculiarities, we extended the former studies with more recent photometric data covering the last 25 years. Our aim was to gain a better insight into the effect of orbital revolution on the pulsation period.

\section{Observational data}

In order to determine the temporal variation of the pulsation period, photometric data that have been acquired after the last extensive study (Vinkó et al. 1993) were collected and analysed. The complete dataset contains measurements from All Sky Automated Survey (ASAS, Pojmanski 2002), All Sky Automated Survey for Supernovae (ASAS-SN, Shappee et al. 2014), Hipparcos (Perryman et al. 1997), Kamogata Sky Survey (KWS, Morokuma et al. 2014) and Gaia (Gaia Collaboration 2016, 2018).

Table 1 contains information about the temporal coverage of the photometric surveys and number of observations analysed in this paper. In addition, $B V$ photometric measurements obtained with the $50 \mathrm{~cm}$ Cassegrain telescope of the
Table 1 Temporal information of the various surveys used for the analysis

\begin{tabular}{llr}
\hline Source & HJD interval & $N$ \\
\hline ASAS & $2452754-2455157$ & 364 \\
ASAS-SN & $2456389-2458380$ & 1074 \\
Hipparcos & $2447889-2448973$ & 75 \\
KWS & $2455752-2458360$ & 509 \\
Gaia & $2457164-2457390$ & 14 \\
\hline
\end{tabular}

Piszkéstetô Mountain Station of the Konkoly Observatory by one of us (L. Sz.) between 1994 and 2007 were also involved in our study. Between 1994 and 1998, an integrating photoelectric photometer equipped with an unrefrigerated EMI 9058QB photomultiplier tube was attached to the telescope, while from the year 2000 on an electrically cooled (to $-20^{\circ} \mathrm{C}$ ) photon counting photometer containing an EMI 9203QB photomultiplier was mounted in the Cassegrain focus.

Both photometers were equipped with standard filters of the Johnson photometric system. The brightness of AU Peg was observed using BD $+17^{\circ} 4575$ as the comparison star (SIMBAD magnitudes: $V=9.24 \mathrm{mag} ; B-V=1.13 \mathrm{mag}$ ), and $\mathrm{BD}+18^{\circ} 4788$ served as the check star. Table 2 contains these previously unpublished measurements obtained in the Piszkéstetô Mountain Station.

Radial velocity (RV) measurement data have also been collected from the literature. In addition to the measurements made by Harris et al. (1984), three additional sets of RV data have been available: those obtained by Barnes et al. (1988), Gorynya et al. (1998) and Vinkó et al. (1998).

\section{Analysis of the photometric data}

Three different approaches have been used to obtain the period length of the pulsation for different time intervals: the discrete Fourier transformation (DFT, Deeming 1975), for which we have used the Period04 analysis software (Lenz and Breger 2005), the phase dispersion minimization (PDM, Stellingwerf 1978) and the method of $O-C$ diagrams (Sterken 2005). Since the first two methods require a constant or slowly varying pulsation period and for the construction of the $O-C$ diagram we would need the correct determination of the phase, e.g. the moments of a chosen phase $(O)$ and the elapsed number of cycles $(E)$, which would become uncertain in the case of strong period variation, the collected data had to be divided into shorter time intervals. The demonstration of this problem can be seen in Fig. 1, where the Fourier amplitude diagrams of the entire ASAS data set of AU Peg is presented. The highest peak on the upper panel corresponds to the pulsation period of 
Table $2 B V$ photometric data obtained with the $50 \mathrm{~cm}$ Cassegrain telescope at Piszkéstetô Mountain Station of the Konkoly Observatory

\begin{tabular}{|c|c|c|c|c|c|}
\hline $\mathrm{JD}-2400000$ & $V$ & $B-V$ & JD-2 400000 & $V$ & $B-V$ \\
\hline 49538.451 & 9.260 & 1.060 & 51757.390 & 9.350 & 0.960 \\
\hline 49569.531 & 9.240 & 0.910 & 51758.373 & 9.080 & 0.840 \\
\hline 49570.415 & 9.370 & 0.930 & 51759.368 & 9.170 & 1.150 \\
\hline 49606.326 & 9.390 & 0.910 & 51782.343 & 9.080 & 0.820 \\
\hline 49606.397 & 9.380 & 0.900 & 51838.266 & 9.120 & 0.900 \\
\hline 49630.240 & 9.390 & 0.950 & 51839.245 & 9.400 & 1.080 \\
\hline 49630.332 & 9.370 & 0.980 & 51840.265 & 9.070 & 0.820 \\
\hline 49631.235 & 9.080 & 0.750 & 51878.264 & 9.280 & 0.900 \\
\hline 49631.299 & 9.070 & 0.760 & 52147.372 & 9.270 & 0.960 \\
\hline 49666.208 & 9.270 & 0.920 & 52150.512 & 9.350 & 0.940 \\
\hline 49666.259 & 9.290 & 0.990 & 52151.335 & 9.070 & 0.850 \\
\hline 49688.211 & 9.360 & 1.010 & 52194.372 & 9.110 & 0.810 \\
\hline 49690.200 & 9.210 & 0.950 & 52195.299 & 9.170 & 0.970 \\
\hline 49900.448 & 9.330 & 1.000 & 52196.292 & 9.360 & 0.930 \\
\hline 49918.413 & 9.080 & 0.790 & 52197.283 & 9.090 & 0.850 \\
\hline 49919.431 & 9.330 & 0.920 & 52198.295 & 9.350 & 0.980 \\
\hline 49920.425 & 9.230 & 0.760 & 52199.336 & 9.050 & 0.810 \\
\hline 49921.428 & 9.150 & 0.960 & 52200.265 & 9.200 & 0.950 \\
\hline 49952.412 & 9.120 & 0.820 & 52589.239 & 9.380 & 0.960 \\
\hline 49986.433 & 9.060 & 0.930 & 52618.265 & 9.390 & 0.920 \\
\hline 49987.313 & 9.350 & 0.990 & 52619.199 & 9.070 & 0.850 \\
\hline 49992.273 & 9.430 & 0.930 & 52620.209 & 9.220 & 0.920 \\
\hline 50015.263 & 9.070 & 0.880 & 52901.384 & 9.070 & 0.860 \\
\hline 50016.285 & 9.400 & 0.970 & 52902.330 & 9.300 & 0.980 \\
\hline 50018.263 & 9.270 & 0.980 & 52903.349 & 9.130 & 0.850 \\
\hline 50338.429 & 9.100 & 0.950 & 52904.313 & 9.160 & 0.950 \\
\hline 50371.274 & 9.220 & 0.830 & 52905.395 & 9.350 & 0.950 \\
\hline 50376.366 & 9.090 & 0.780 & 52906.316 & 9.080 & 0.860 \\
\hline 50605.476 & 9.100 & 0.820 & 52947.226 & 9.050 & 0.840 \\
\hline 50633.482 & 9.320 & 1.000 & 52947.303 & 9.060 & 0.860 \\
\hline 50634.425 & 9.130 & 0.770 & 52948.225 & 9.300 & 0.980 \\
\hline 50749.268 & 9.330 & 0.990 & 52948.304 & 9.350 & 1.000 \\
\hline 50749.333 & 9.400 & 0.920 & 53266.337 & 9.290 & 0.980 \\
\hline 50750.274 & 9.110 & 0.790 & 53267.328 & 9.130 & 0.850 \\
\hline 50750.333 & 9.110 & 0.780 & 53286.317 & 9.290 & 0.910 \\
\hline 50751.246 & 9.150 & 0.960 & 53331.235 & 9.270 & 0.990 \\
\hline 50751.308 & 9.180 & 0.910 & 53569.430 & 9.100 & 0.890 \\
\hline 51051.396 & 9.190 & 0.820 & 53612.350 & 9.070 & 0.800 \\
\hline 51052.363 & 9.140 & 0.920 & 53614.347 & 9.280 & 0.890 \\
\hline 51080.300 & 9.200 & 0.810 & 54389.298 & 9.110 & 0.830 \\
\hline 51756.463 & 9.180 & 0.920 & 54390.270 & 9.270 & 0.970 \\
\hline
\end{tabular}

$P_{\text {pul }}=2.4122$ days. The lower panel is obtained from the residuals after the fitting of the first frequency. The highest peak in this diagram corresponds to $P=2.4147$ days. From the proximity of these period values we assumed that

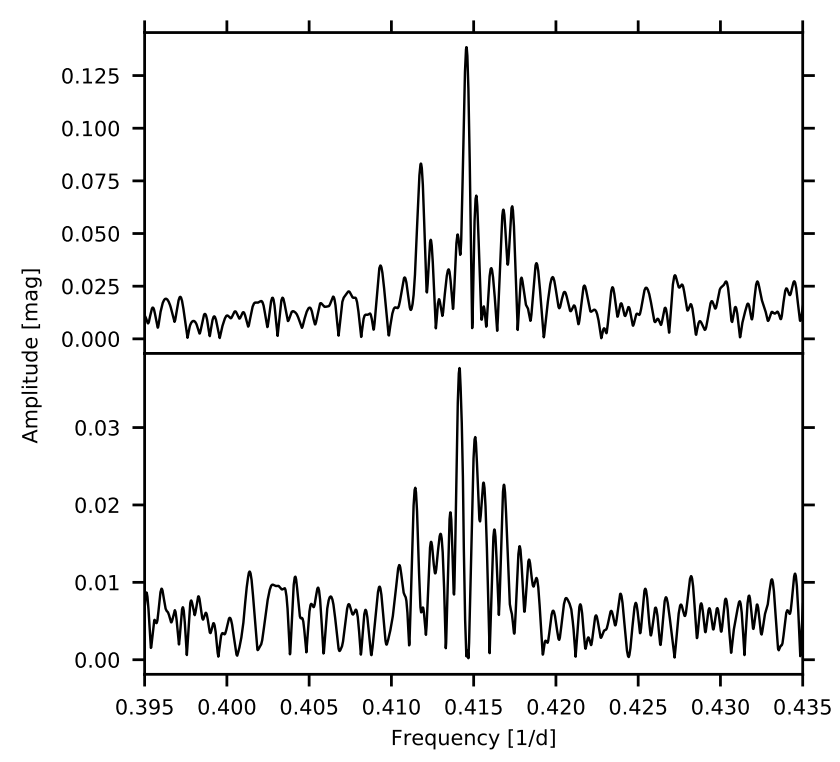

Fig. 1 The Fourier spectrum of the original ASAS data (top panel) and that of the residual data, after subtracting the main frequency (bottom panel)

the Cepheid underwent a rapid period variation in the time interval covered by the ASAS observations.

Since the period variation covered by the collected data was not strong enough for the phase shift affecting the moments of light curve extrema to accumulate into a longer time than the period itself, we decided to create the $O-C$ diagram for these measurements. As a first step of the analysis, every set of observation has been split into smaller subsets. For each survey, 250 day long temporal bins were defined, in which each datapoint was moved to a new subset. The phase curves of each previously created subset of measurements have been calculated, which then were used to determine the observed $(O)$ epoch values. This method inevitably decreases the resolution of the resulting $O-C$ curve, but the precision of the results increases, since the error of the phase calculation will decrease significantly. The $O-C$ diagram of $V$ band measurements was calculated assuming the elements:

$C=\mathrm{JD} 2453481.812+2.412^{\mathrm{d}} \cdot E$.

The obtained $O-C$ values are listed in Table 4, while the corresponding diagram is presented in Fig. 2.

It has been found that the segments of the $O-C$ graph can be described with linear functions, thus the pulsation period of the Cepheid remains approximately the same for the different time intervals. The change of the pulsation period shown by the ASAS data can be approximated as a parabolic function on the $O-C$ diagram, hence it can be described as a linear period change (see later in this chapter). Table 3 describes the linear fits in the different time intervals. As illustrated in Fig. 3, it has been found that the $O-C$ data points 


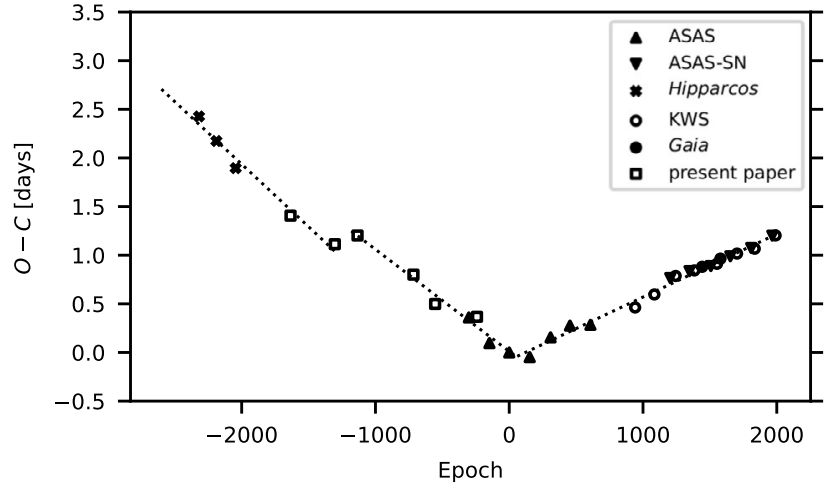

Fig. 2 The $O-C$ diagram of AU Pegasi calculated from the new measurements with linear fit segments

Table 3 The fitted lines for the different time intervals (see Fig. 2), and the calculated periods

\begin{tabular}{llrr}
\hline Epoch & \multicolumn{2}{l}{ Linear fit } & Period [d] \\
\hline$-2600<E<-1280$ & $-0.0013 \cdot E-0.0636$ & 2.4109 \\
& $\pm 0.00013 \quad \pm 0.0851$ & \pm 0.0001 \\
$-1180<E<60$ & $-0.0010 \cdot E+0.0108$ & 2.4111 \\
& \pm 0.00014 & \pm 0.0562 & \pm 0.0001 \\
$60<E<2000$ & $0.0006 \cdot E-0.0780$ & 2.4128 \\
& \pm 0.00002 & \pm 0.0243 & \pm 0.0001 \\
\hline
\end{tabular}

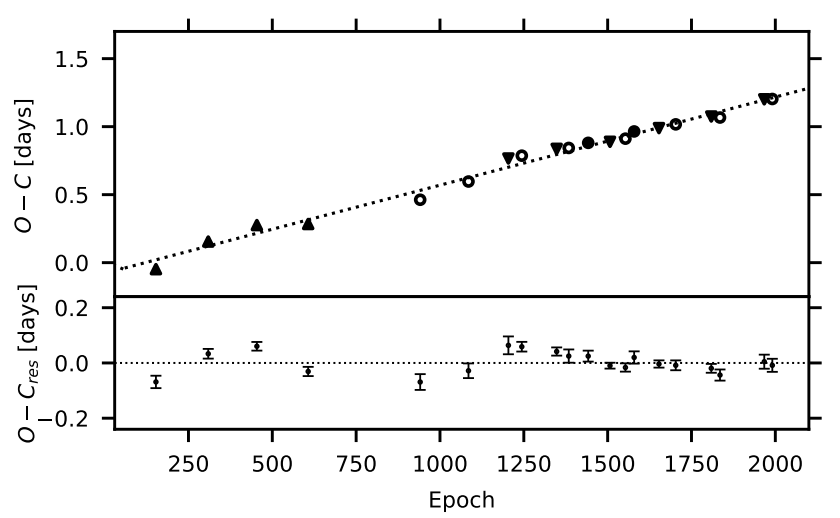

Fig. 3 Top panel: The last linear segment of the $O-C$ diagram and its linear fit. Bottom panel: The residual values of the $O-C$ diagram after subtracting the linear fit. The notation of the data points is the same as in Fig. 2

obtained from ASAS, ASAS-SN and KWS measurements deviate from the fitted linear curve in a periodic manner.

The period of this cyclic behaviour is approximately 2215 days, while its amplitude is 0.05 days. This variation cannot originate from the light-time effect caused by the known companion of the Cepheid, since the expected amplitude of this variation would be as low as 0.001 days, and the period is not appropriate, either. To examine whether the obtained periodic variation could correspond to the light-time

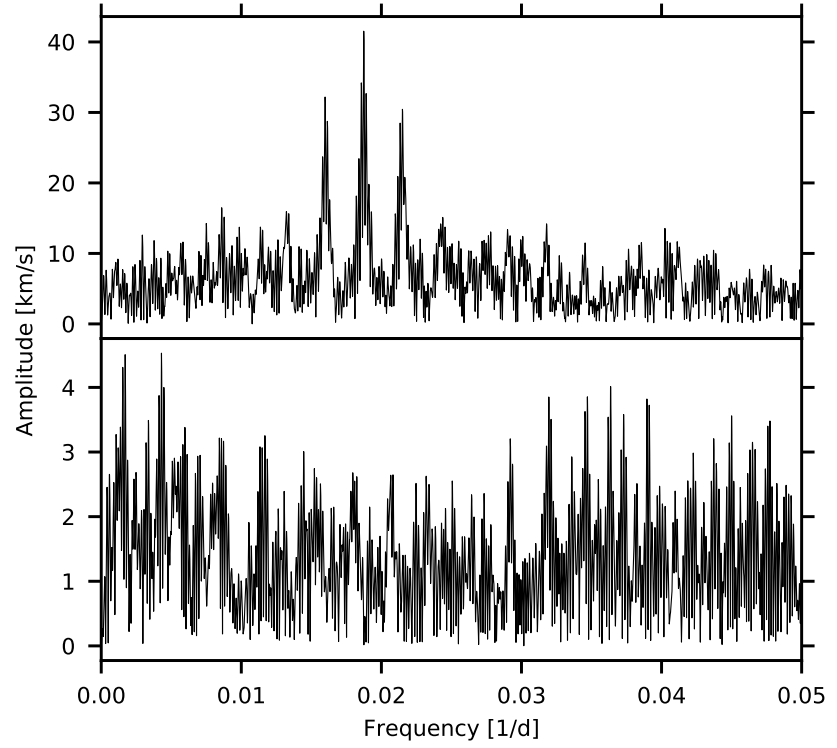

Fig. 4 The Fourier spectrum obtained from the RV measurements (top panel) and that of the residual data after whitening with the frequency of the known orbital motion

effect of a formerly unknown companion, we analysed the available RV observations collected from the literature. The Fourier spectra of the RV observations before and after subtracting the main frequency (the known orbital motion) are presented in Fig. 4. Since the expected RV projection corresponding to the obtained period and amplitude of the variation is $45.7 \mathrm{~km} / \mathrm{s}$ (assuming circular orbit), which would then result in a sharp peak in the Fourier diagram of the RV observations at the frequency of $4.515 \cdot 10^{-4}$ cycles/day, that is clearly not present (although the Fourier spectrum shows a peak with a much smaller amplitude at that frequency), we cannot attribute the observed variation to any effect caused by the orbital motion of the Cepheid.

In the case of most archival photometric data series and the measurements presented in this paper as well, not only $V$ band, but $B$ band observations were also available. With the use of available $B$ band data, another set of $O$ values has been calculated. Since these measurements covered the time interval in which a rapid period increase was observed (Vinkó et al. 1993), the $O-C$ diagram of $B$ band could not have been created, but the simultaneous $V$ and $B$ observations allowed the comparison of $B$ and $V$ band epochs. The differences of the same phase $O$ values calculated from $B$ and $V$ band observations are presented in Fig. 5. Table 5 contains the calculated differences of the two sets of $O$ values. According to the results, the brightness maximum in the $B$ band light curve precedes the $V$ band with approximately 0.082 days $(\Delta \phi=0.039$ for the phase shift). This corresponds well to the former observations (Freedman 1988), where a systematic phase shift was found for several Cepheids, which appeared to be increasing for longer wavelengths. All photometric measurements 
Table $4 O-C$ values of AU Peg calculated from the seasonal $V$ band datasets of the different surveys

\begin{tabular}{lrrll}
\hline HJD-2400 000 & \multicolumn{1}{l}{ E } & $\begin{array}{l}O-C \\
(\mathrm{~d})\end{array}$ & $\begin{array}{l}\sigma \\
(\mathrm{d})\end{array}$ & Survey \\
\hline 47888.061 & -2319 & 2.427 & 0.016 & Hipparcos \\
48201.388 & -2189 & 2.174 & 0.020 & Hipparcos \\
48548.458 & -2045 & 1.895 & 0.019 & Hipparcos \\
49536.949 & -1635 & 1.407 & 0.031 & present paper \\
49898.720 & -1485 & 1.356 & 0.032 & present paper \\
51755.516 & -716 & 0.800 & 0.060 & present paper \\
52145.983 & -554 & 0.499 & 0.039 & present paper \\
52753.705 & -302 & 0.360 & 0.021 & ASAS \\
52900.852 & -241 & 0.367 & 0.043 & present paper \\
53124.911 & -148 & 0.096 & 0.026 & ASAS \\
53481.812 & 0 & 0.000 & 0.014 & ASAS \\
53850.823 & 153 & -0.048 & 0.022 & ASAS \\
54227.321 & 309 & 0.155 & 0.018 & ASAS \\
54577.203 & 454 & 0.276 & 0.015 & ASAS \\
54946.269 & 607 & 0.284 & 0.016 & ASAS \\
55752.104 & 941 & 0.462 & 0.028 & KWS \\
56099.587 & 1085 & 0.597 & 0.026 & KWS \\
56386.801 & 1204 & 0.766 & 0.032 & ASAS-SN \\
56483.308 & 1244 & 0.787 & 0.018 & KWS \\
56734.221 & 1348 & 0.837 & 0.015 & ASAS-SN \\
56821.065 & 1384 & 0.843 & 0.024 & KWS \\
56961.008 & 1442 & 0.881 & 0.019 & Gaia \\
57117.804 & 1507 & 0.888 & 0.011 & ASAS-SN \\
57228.786 & 1553 & 0.912 & 0.015 & KWS \\
57291.555 & 1579 & 0.965 & 0.020 & Gaia \\
57470.078 & 1653 & 0.989 & 0.013 & ASAS-SN \\
57590.713 & 1703 & 1.067 & 0.018 & KWS \\
57846.458 & 1809 & 1.074 & 0.025 & ASAS-SN \\
57909.167 & 1835 & 1.067 & 0.020 & KWS \\
58227.704 & 1967 & 1.201 & 0.024 & ASAS-SN \\
58285.598 & 1991 & 1.203 & 0.024 & KWS \\
\hline & & & &
\end{tabular}

have also been analysed with the DFT and PDM methods. To prevent the mixing of different period values, observation subsets shorter than 250 days were created. Data from different surveys were treated separately. For the PDM method parameters $N_{\mathrm{b}}=10$ and $N_{\mathrm{c}}=3$ have been used, where $N_{\mathrm{b}}$ and $N_{\mathrm{c}}$ denote the number of bins and the number of covers (Stellingwerf 1978).

Some of the datasets, like the ASAS-SN observations, proved to contain significant number of outliers and exhibit a higher scatter in the data, which would result in less precise period evaluation. To address this problem during the PDM fit, the outlying data points deviating from the calculated phase curve with more than $2 \sigma$ were excluded, and the phase curve was calculated again. We have tested the

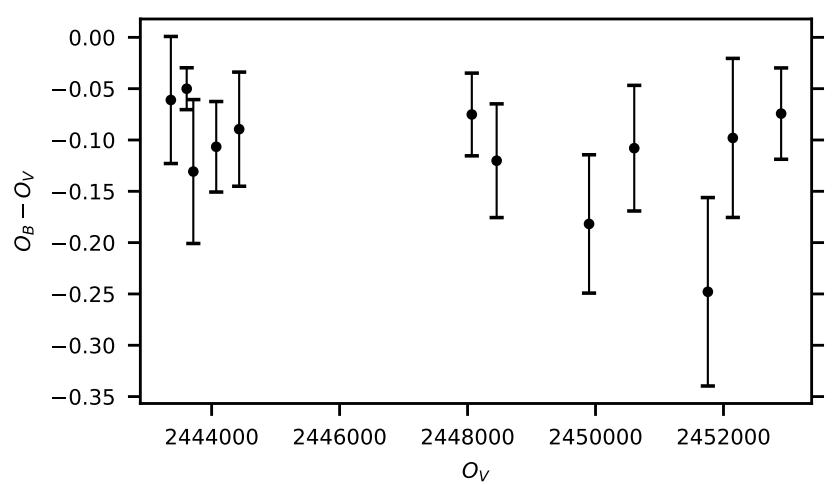

Fig. 5 The differences of the $O$ values in $B$ and $V$ bands in days as a function of the Julian Day

Table 5 The time difference of the moments of brightness maxima and its standard deviation $(\sigma)$ in different bands in days

\begin{tabular}{llll}
\hline HJD-2 400000 & $O_{\mathrm{B}}-O_{\mathrm{V}}$ & $\sigma$ & Reference \\
\hline 43362.532 & -0.061 & 0.062 & Vinkó et al. (1993) \\
43610.115 & -0.050 & 0.021 & Henden (1980) \\
43713.625 & -0.131 & 0.070 & Vinkó et al. (1993) \\
44071.742 & -0.107 & 0.044 & Moffett and Barnes (1984) \\
44430.211 & -0.089 & 0.056 & Moffett and Barnes (1984) \\
48066.403 & -0.075 & 0.041 & Vinkó et al. (1993) \\
48454.503 & -0.120 & 0.055 & Vinkó et al. (1993) \\
49898.720 & -0.182 & 0.067 & present paper \\
50605.330 & -0.108 & 0.061 & present paper \\
51755.516 & -0.248 & 0.092 & present paper \\
52145.983 & -0.098 & 0.078 & present paper \\
52900.852 & -0.074 & 0.045 & present paper \\
\hline
\end{tabular}

method with different thresholds and the $2 \sigma$ cut appeared to be the best choice for an automated outlier removal: with the $1 \sigma$ threshold many points were removed that could not have been flagged as definite outliers, while at $3 \sigma$ not all outliers were removed. An example for the $V$ band phase curve of the Cepheid is presented in Fig. 6.

Table 6 contains the calculated period values for the different surveys and various methods. The period has been calculated with every method, if the temporal coverage and the amount of data points in the seasonal subset allowed it. For the ASAS, ASAS-SN and KWS the period has been calculated with every method. In the case of the Gaia data, the amount of measurements and the short term coverage allowed the use of DFT, while the number of data points was insufficient to calculate the pulsation period properly with PDM. Since the time interval of the Gaia photometric data was covered by ASAS-SN and KWS observations, the period was calculated with the help of $O-C$ method as well. The photometric data obtained by Hipparcos had to be treated differently, since the measurements covered only 


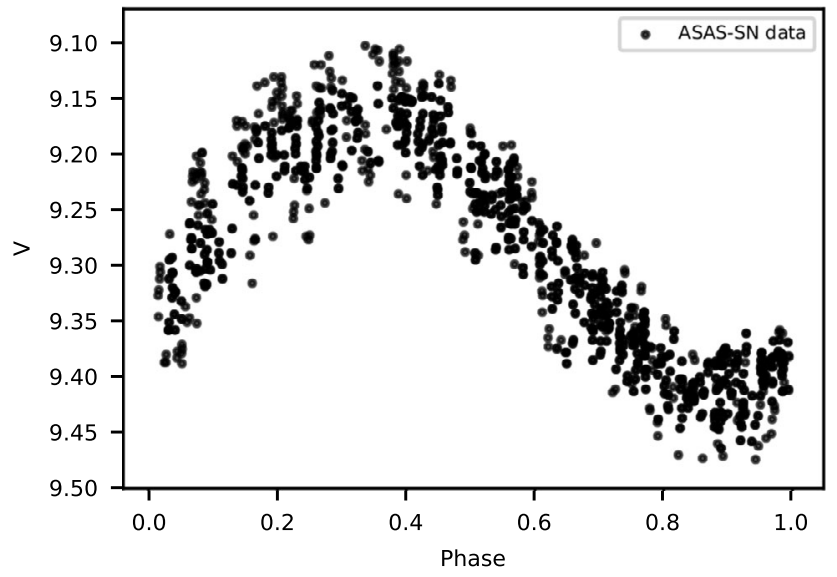

Fig. 6 Light curve in V calculated from the ASAS-SN data. The phases were calculated using the periods listed in Table 6

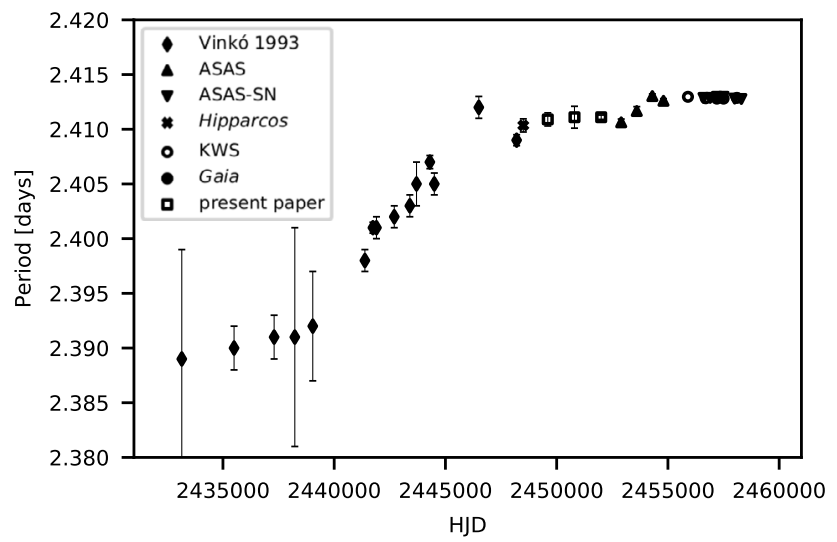

Fig. 7 The pulsation period of AU Pegasi as a function of time

short time, but the amount of data points was larger than in the case of Gaia measurements. This time interval was not covered by any other surveys, thus the pulsation period was only calculated with DFT and PDM for this survey. In the case of the data obtained at Piszkéstetô Observatory, the measurements were scattered in time and covered several years. For this reason, the pulsation period was only calculated with $O-C$ method. The final period value was the average of the pulsation periods obtained with the different methods for every survey (Table 6). The pulsation period of AU Peg is presented as a function of time in Fig. 7.

According to the Hipparcos and Piszkéstetô measurements, the pulsation period of AU Pegasi was slightly increasing between HJD 2448500 and 2452000 at a rate of $8.348 \cdot 10^{-5}$ day/year. Between HJD 2452900 and 2454850 the rate of the period change increased according to the ASAS measurements, to the value of $3.746 \cdot 10^{-4}$ day/year, which is approximately half of the rate the pulsation period had been changing with between HJD 2442500 and 2446000 . After this rapid change, the period seemed to keep its value, and it remained constant until the latest
Table 6 The pulsational period values calculated from the seasonal data of different surveys. $T$ : HJD-2 $400000 ; P$ : the obtained period in days

\begin{tabular}{|c|c|c|c|c|c|}
\hline Survey & $T$ & $\mathrm{P}_{\mathrm{DFT}}$ & $\mathrm{P}_{\text {PDM }}$ & $\mathrm{P}_{O-C}$ & $\mathrm{P}_{\text {final }}$ \\
\hline \multirow[t]{8}{*}{ ASAS } & \multirow[t]{2}{*}{52900} & 2.4103 & 2.4105 & 2.4111 & 2.4106 \\
\hline & & \pm 0.0002 & \pm 0.0001 & \pm 0.0001 & \pm 0.0003 \\
\hline & \multirow[t]{2}{*}{53600} & 2.4119 & 2.4119 & 2.4111 & 2.4117 \\
\hline & & \pm 0.0001 & \pm 0.0001 & \pm 0.0001 & \pm 0.0003 \\
\hline & \multirow[t]{2}{*}{54300} & 2.4133 & 2.4130 & 2.4128 & 2.4130 \\
\hline & & \pm 0.0001 & \pm 0.0002 & \pm 0.0001 & \pm 0.0002 \\
\hline & \multirow[t]{2}{*}{54800} & 2.4124 & 2.4126 & 2.4128 & 2.4126 \\
\hline & & \pm 0.0009 & \pm 0.0003 & \pm 0.0001 & \pm 0.0004 \\
\hline \multirow[t]{12}{*}{ ASAS-SN } & \multirow[t]{2}{*}{56600} & 2.4128 & 2.4130 & 2.4128 & 2.4129 \\
\hline & & \pm 0.0007 & \pm 0.0003 & \pm 0.0001 & \pm 0.0002 \\
\hline & \multirow[t]{2}{*}{56900} & 2.4128 & 2.4130 & 2.4128 & 2.4129 \\
\hline & & \pm 0.0008 & \pm 0.0002 & \pm 0.0001 & \pm 0.0002 \\
\hline & \multirow[t]{2}{*}{57200} & 2.4129 & 2.4132 & 2.4128 & 2.4130 \\
\hline & & \pm 0.0004 & \pm 0.0001 & \pm 0.0001 & \pm 0.0002 \\
\hline & \multirow[t]{2}{*}{57500} & 2.4127 & 2.4134 & 2.4128 & 2.4130 \\
\hline & & \pm 0.0002 & \pm 0.0002 & \pm 0.0001 & \pm 0.0002 \\
\hline & \multirow[t]{2}{*}{58000} & 2.4126 & 2.4130 & 2.4128 & 2.4128 \\
\hline & & \pm 0.0009 & \pm 0.0002 & \pm 0.0001 & \pm 0.0002 \\
\hline & \multirow[t]{2}{*}{58300} & 2.4127 & 2.4128 & 2.4128 & 2.4128 \\
\hline & & \pm 0.0004 & \pm 0.0001 & \pm 0.0001 & \pm 0.0001 \\
\hline \multirow[t]{2}{*}{ Gaia } & \multirow[t]{2}{*}{57200} & 2.4128 & - & 2.4128 & 2.4128 \\
\hline & & \pm 0.0003 & & \pm 0.0001 & \pm 0.0001 \\
\hline \multirow[t]{2}{*}{ Hipparcos } & \multirow[t]{2}{*}{48500} & 2.4103 & 2.4104 & - & 2.4104 \\
\hline & & \pm 0.0002 & \pm 0.0001 & & \pm 0.0001 \\
\hline \multirow[t]{8}{*}{ KWS } & \multirow[t]{2}{*}{55900} & 2.4131 & 2.4130 & 2.4128 & 2.4130 \\
\hline & & \pm 0.0002 & \pm 0.0001 & \pm 0.0001 & \pm 0.0001 \\
\hline & \multirow[t]{2}{*}{56700} & 2.4127 & 2.4130 & 2.4128 & 2.4128 \\
\hline & & \pm 0.0003 & \pm 0.0001 & \pm 0.0001 & \pm 0.0002 \\
\hline & \multirow[t]{2}{*}{57500} & 2.4127 & 2.4129 & 2.4128 & 2.4128 \\
\hline & & \pm 0.0003 & \pm 0.0001 & \pm 0.0001 & \pm 0.0001 \\
\hline & \multirow[t]{2}{*}{58100} & 2.4129 & 2.4129 & 2.4128 & 2.4129 \\
\hline & & \pm 0.0002 & \pm 0.0002 & \pm 0.0001 & \pm 0.0001 \\
\hline \multirow{6}{*}{$\begin{array}{l}\text { Present } \\
\text { paper }\end{array}$} & \multirow[t]{2}{*}{49600} & 2.4110 & - & 2.4109 & 2.4109 \\
\hline & & \pm 0.0021 & & \pm 0.0001 & \pm 0.0006 \\
\hline & \multirow[t]{2}{*}{50800} & 2.4113 & - & 2.4111 & 2.4111 \\
\hline & & \pm 0.0033 & & \pm 0.0001 & \pm 0.0010 \\
\hline & \multirow[t]{2}{*}{52000} & 2.4112 & - & 2.4111 & 2.4111 \\
\hline & & \pm 0.0002 & & \pm 0.0001 & \pm 0.0002 \\
\hline
\end{tabular}

observations. This behaviour has not been observed before and it is still an open question, how the companion of the Cepheid affects the pulsation, and if there is a direct connection between the evolution of the period and binarity of the star. 


\section{Revised spectroscopic orbit of AU Peg}

The orbital elements of the binary system involving AU Peg have been determined by Harris et al. (1984) based on their own RV data. When revising the elements of the spectroscopic orbit, the available RV data (i.e. those published by Harris et al. (1984), Barnes et al. (1988), Gorynya et al. (1998) and Vinkó et al. (1998)) were split into subsets covering no more than two years. These data sets then have been corrected for variations due to the pulsation by fitting and subtracting the sinusoidal changes corresponding to the pulsation period and its first harmonic. The amplitude ratio of the two fitted components is 10:1. The second harmonic can be neglected, since its amplitude is not large enough to make the signal distinguishable from the noise in the Fourier spectrum. Since the pulsation period of the Cepheid was changing significantly during and between the different RV measurements, we used the diagram shown in Fig. 7 to obtain the correct value for the pulsation period. While subtracting the contribution of the first harmonic from the RV data, we assumed that the RV amplitude ratio of the fundamental and first harmonic variation is the same as in the case of the light curve. To obtain the orbital parameters we fitted

$v_{i}=V-K\left(\cos \left(f_{i}+\bar{\omega}\right)+e \cos \bar{\omega}\right)$

to the pulsation corrected dataset, where $v_{i}$ denotes the $i$ th RV entry corresponding to $f_{i}$ true anomaly at time entry $t_{i}$. In the formula above, $V$ is the systemic velocity of the system, $K$ is the semi-amplitude of the variation, $e$ is the eccentricity of the orbit, and $\bar{\omega}$ is the argument of the periapsis (Fulton et al. 2018). To calculate the mean anomalies at various time entries we also had to fit the periastron passage factor $\chi$, which is the fraction of orbit prior to the start of data-taking that periastron occurred.

We used a Bayesian approach to fit the RV data and extracted the orbital parameters along with their uncertainties using Markov Chain Monte Carlo (MCMC) simulations. To implement this method we have used the radvel python package introduced and described in Fulton et al. (2018). The prior distributions were chosen to be uniform centered on the parameters obtained in Harris et al. (1984), except for the eccentricity and the longitude of the periastron, for which every possible value was considered. The obtained fit along with the orbital RV phase curve is presented in Fig. 8.

The computed elements are

$$
\begin{aligned}
P & =53.3344 \pm 0.0003 \mathrm{~d} \\
V & =-1.6368 \pm 0.0004 \mathrm{~km} / \mathrm{s} \\
K & =44.091 \pm 0.592 \mathrm{~km} / \mathrm{s} \\
e & =0.0425 \pm 0.0027 \\
\chi & =0.3264 \pm 0.0005
\end{aligned}
$$

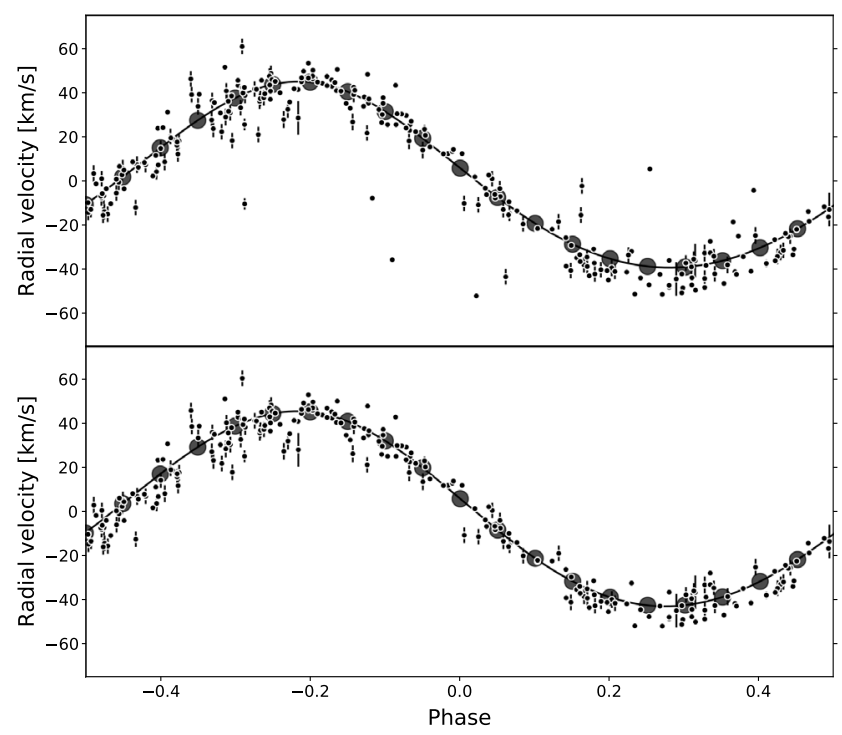

Fig. 8 The orbital RV curve after correcting for the pulsation and the obtained fit. Top panel: the obtained fit with the discrepant datapoints included. Bottom panel: the fit obtained after removing the these points

$\bar{\omega}=0.3404 \pm 0.0040 \mathrm{rad}$.

We have compared these orbital elements to those obtained in Harris et al. (1984). According to our study, the orbit is fairly different from the previously assumed one: the orbital period appears to be longer than obtained before ( $53.319 \pm 0.015$ days) and the calculated amplitude of variation is larger by $2.2 \mathrm{~km} / \mathrm{s}$ than the previous one, as well. The eccentricity of the orbit appears to be smaller, thus according to our calculations, the orbit itself is more circular, than it was originally believed ( $e=0.12 \pm 0.04$, Harris et al. 1984). It has been mentioned in Harris et al. (1984), that by omitting a discrepant point from their dataset, they obtained an orbit with smaller eccentricity, which is more similar to the solution we obtained. Since for the solution above we discarded all discrepant points (through creating the phase curve in every 250 day long time interval supposing a sinusoidal change, then removing the outliers with the help of the previously shown $2 \sigma$ clipping) we tested whether the orbit obtained from the original data, including the previously deleted points, would be more similar to the one in Harris et al. (1984). In this case the period remained the same, but the amplitude decreased by $2 \mathrm{~km} / \mathrm{s}$, thus its value became very similar to that obtained in Harris et al. (1984). The eccentricity of the orbit turned out to be larger than in the first case ( $e=0.068 \pm 0.004)$ due to these discrepant datapoints. Although this value is within the error limits given in Harris et al. (1984), it still corresponds to a more circular orbit and we believe, that omitting the discrepant datapoints is a reasonable choice, considering their high scatter from the fitted phase curve (Fig. 8). 


\section{Summary}

It has been presented that, in contrast to the previous behaviour of AU Pegasi, the rate of pulsation period change has decreased significantly and the period has come to a constant value, according to the latest observations. The last data point from the analysis of Vinkó et al. (1993) suggests that there might have been another time interval (between JD 2445000 and 2447500), when the pulsation period set in a constant value over time, followed by a rapid period change. If this behaviour turns out to be periodic, it might be an indicator for the interaction between the Cepheid and its companion.

According to our analysis, a wave in the $O-C$ diagram has been found, which could not have been connected to any known physical process in the environment of the Cepheid. The amplitude and period of this variation might correspond well to light-time effect, but the lack of this periodicity in the RV data rules this possibility out. This effect might be the result of the tidal interaction between the Cepheid and its companion, but to support this hypothesis, further observations and analysis would be required.

We have also revised the spectroscopic orbit of AU Peg. By subtracting the contributions of the pulsation from the RV data taking into account the strong changes observed in the pulsation period (see Fig. 7) and by using Bayesian framework for the fitting, we could reliably determine the orbital elements of AU Peg. According to our analysis, the orbit of AU Peg is more circular than it was previously determined, regardless how one handles the discrepant datapoints (the eccentricity obtained in the case of the whole dataset is $e=0.068 \pm 0.004$, while after removing the mentioned datapoints the fitting process resulted in $e=0.0425 \pm 0.0027$ ). The resulting amplitude of the RV variation and the orbital period values were larger than the ones obtained in Harris et al. (1984), which, together with the smaller eccentricity, indicate higher mass function for the companion star.

The peculiar behaviour of the pulsation period of AU Pegasi necessitates frequent photometric observations of this interesting binary system with a Type II Cepheid component.

Acknowledgements Open access funding provided by MTA Research Centre for Astronomy and Earth Sciences (MTA CSFK). This project has been supported by the GINOP-2.3.2-15-2016-00003 grant of the Hungarian National Research, Development and Innovation Office (NKFIH). This work has been partly supported by the Lendület Program of the Hungarian Academy of Sciences, project No. LP20187/2018 and the Hungarian NKFIH projects K-115 709 and K-129 249.

Publisher's Note Springer Nature remains neutral with regard to jurisdictional claims in published maps and institutional affiliations.
Open Access This article is distributed under the terms of the Creative Commons Attribution 4.0 International License (http://creative commons.org/licenses/by/4.0/), which permits unrestricted use, distribution, and reproduction in any medium, provided you give appropriate credit to the original author(s) and the source, provide a link to the Creative Commons license, and indicate if changes were made.

\section{References}

Antipin, S.V., Sokolovsky, K.V., Ignatieva, T.I.: Mon. Not. R. Astron. Soc. 379, 60 (2007)

Barnes, I.T.G., Moffett, T.J., Slovak, M.H.: Astrophys. J. Suppl. Ser. 66, 43 (1988)

Benedict, G.F., McArthur, B.E., Feast, M.W., Barnes, T.G., Harrison, T.E., Patterson, R.J., Menzies, J.W., Bean, J.L., Freedman, W.L.: Astron. J. 133, 1810 (2007)

Deeming, T.J.: Astrophys. Space Sci. 36(1), 137 (1975)

Erleksova, G.E.: Perem. Zvezdy 21, 97 (1978)

Freedman, W.L.: Astrophys. J. 326, 691 (1988)

Fulton, B.J., Petigura, E.A., Blunt, S., Sinukoff, E.: Publ. Astron. Soc. Pac. 130, 044504 (2018)

Gaia Collaboration, Prusti, T., de Bruijne, J.H.J., Brown, A.G.A., Vallenari, A., Babusiaux, C., Bailer-Jones, C.A.L., Bastian, U., Biermann, M., Evans, D.W., et al.: Astron. Astrophys. 595, 1 (2016)

Gaia Collaboration, Brown, A.G.A., Vallenari, A., Prusti, T., de Bruijne, J.H.J., Babusiaux, C., Bailer-Jones, C.A.L., Biermann, M., Evans, D.W., Eyer, L., et al.: Astron. Astrophys. 616, 1 (2018)

Gorynya, N.A., Samus', N.N., Sachkov, M.E., Rastorguev, A.S., Glushkova, E.V., Antipin, S.V.: Astron. Lett. 24, 815 (1998)

Groenewegen, M.A.T.: Astron. Astrophys. 619, 8 (2018)

Harris, H.C., Welch, D.L.: Astron. J. 98, 981 (1989)

Harris, H., Olszewski, E.W., Wallerstein, G.: Astron. J. 84, 1598 (1979)

Harris, H.C., Olszewski, E.W., Wallerstein, G.: Astron. J. 89, 119 (1984)

Henden, A.A.: Mon. Not. R. Astron. Soc. 192, 621 (1980)

Kovtyukh, V., Yegorova, I., Andrievsky, S., Korotin, S., Saviane, I., Lemasle, B., Chekhonadskikh, F., Belik, S.: Mon. Not. R. Astron. Soc. 477, 2276 (2018)

Lenz, P., Breger, M.: Commun. Asteroseismol. 146, 53 (2005)

Maas, T., Giridhar, S., Lambert, D.L.: Astrophys. J. 666, 378 (2007)

McAlary, C.W., Welch, D.L.: Astron. J. 91, 1209 (1986)

Moffett, T.J., Barnes, T.G.: Astrophys. J. Suppl. Ser. 55, 389 (1984)

Morokuma, T., Tominaga, N., Tanaka, M., Mori, K., Matsumoto, E., Kikuchi, Y., Shibata, T., Sako, S., Aoki, T., Doi, M., et al.: Publ. Astron. Soc. Jpn. 66(6), 114 (2014)

Perryman, M.A.C., et al.: Astron. Astrophys. 323, 49 (1997)

Pojmanski, G.: Acta Astron. 52, 397 (2002)

Shappee, B., et al.: In: American Astronomical Society Meeting Abstracts \#223. American Astronomical Society Meeting Abstracts, vol. 223 , p. 236 (2014)

Stellingwerf, R.F.: Astrophys. J. 224, 953 (1978)

Sterken, C.: In: Sterken, C. (ed.) The Light-Time Effect in Astrophysics: Causes and Cures of the O-C Diagram. ASP Conf. Ser., vol. 335 , p. 3 (2005)

Szabados, L.: Commun. Konkoly Obs. Hung. 70, 1 (1977)

Vinkó, J., Szabados, L., Szatmáry, K.: Astron. Astrophys. 279, 410 (1993)

Vinkó, J., Evans, N.R., Kiss, L.L., Szabados, L.: Mon. Not. R. Astron. Soc. 296, 824 (1998)

Wallerstein, G.: Publ. Astron. Soc. Pac. 114, 689 (2002) 